MATEC Web of Conferences 11,01017 (2014)

DOI: $10.1051 /$ matecconf $/ 20141101017$

(C) Owned by the authors, published by EDP Sciences, 2014

\title{
Thermal properties of a new ecological building material / Granular cork embedded in white cement
}

\author{
Abou-bakr CHERKI ${ }^{1}$, Abdelhamid KHABBAZI ${ }^{1}$, Soumia MOUNIR ${ }^{1}$ And Youssef MAALOUFA ${ }^{1}$ \\ ${ }^{1}$ Université Mohamed V-Agdal, Ecole Mohamedia d'Ingénieurs, Laboratoire Energétique Matériaux et Environnement, \\ EST-Salé, Maroc
}

\begin{abstract}
Cork, natural and renewable product, has thermal and acoustic properties very interesting because of its microstructure and porosity representing a significant portion of its apparent volume; it's coming from Moroccan Maamora's forest. This work is a contribution to understand the thermal behaviour of the composite material based on granular cork embedded in white cement. An experimental investigation of its thermal properties was mainly performed using the asymmetrical device of transient Hot Plate method. The effect of granular cork size on the thermal properties of the mixture was studied. The experimental study of this sustainable material aims to characterize its thermal properties and then compare them with those of white cement without cork for motivate the proposal that this composite material will be used as walls insulator. A comparison of the energy performances of the composite material and white cement was made; it allows deducing a very interesting energy gain. The findings of the experiments indicate that the composite is better than white cement in term of thermal insulation, energy storage capacity and lightness. So, it can be used to realize the internal walls insulation. Its utilization should contribute to the improvement of the energy efficiency in building especially that this is a mixture based on a sustainable and renewable material.
\end{abstract}

\section{Introduction}

The use of a new thermal insulating material requires prior knowledge of all its thermal properties, especially if it's a natural material, durable and ecologic as the cork. The objective of this paper is to characterize the thermal properties of a new composite material based on granular cork bound with white cement (cf. picture Fig. 1). To do this, authors essentially based this work on the asymmetrical device of transient Hot Plate method [1]. The idea of consolidate granular cork with white cement aims to give us a lighter material and certainly more insulating than white cement without cork, and modify the thermal capacity of white cement by the presence of cork in the composition of the new composite material. Some studies have already been established and published about materials based on granular cork. Khabbazi and al. [2] conducted an experimental hygrothermal study on a new insulating material based on granular cork. Another study of the thermal and mechanical properties of a new material based on granular cork and cement mortar has been realized by Khabbazi et al. [3]. These references show the usefulness of the cork, abundant in Morocco, likely to be a material of choice in thermal insulation applications. Several characterization studies of porous materials have been completed and published using the transient Hot Plate method. Mention that Jannot and al. [4] have used the symmetrical device of this method to characterize the thin insulating materials. Bal and al. [1] have adopted the recent asymmetrical device to characterize the laterite based bricks with millet waste additive material. All this motivated us to adopt this method and apply it to our new material. The characterization of thermal properties of this new composite material will valorise it (comparing its properties with those of white cement) for its utilisation as walls insulator.

\section{Experimental approach and principle of used methods}

\subsection{Samples preparation}

The apparent volume of dimensions 100x100x20 mm was filled by granular cork, and then white cement paste (with a water-white cement ratio of 0.5 by mass) was added for that it occupies, after hardening, the intergranular space and a very thin layer surrounding the granular cork. The use of different size categories of granular cork is realized to verify if the grains size has an effect on thermal properties of the material. So, three samples series corresponding to three different size categories of granular cork were prepared, using a normalized sieving process $\left(\mathrm{d}_{1}-\mathrm{D}_{1}=5-6.3 \mathrm{~mm}, \mathrm{~d}_{2}-\mathrm{D}_{2}=6.3\right.$ $\left.8 \mathrm{~mm}, \mathrm{~d}_{3}-\mathrm{D}_{3}=8-12.5 \mathrm{~mm}\right)$. 


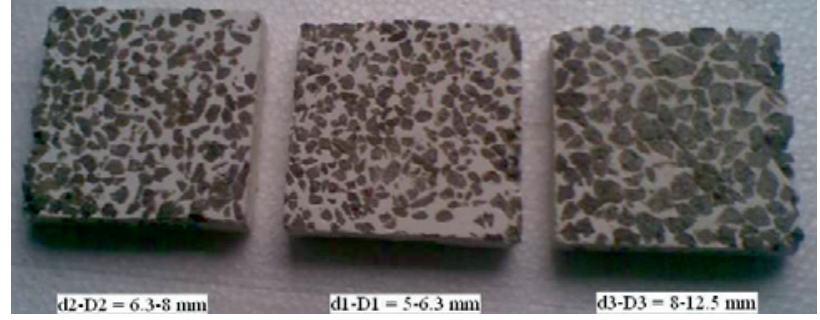

Fig. 1. View of the new composite (cork-white cement).

Furthermore, a sample of white cement without cork was prepared; having the same dimensions as the other three, in order to compare the variation of thermal properties of the mixture, depending on granular cork size, with those of white cement. The four samples are then drawn in a vacuum drying chamber to remove moisture present into the pores of each one. Next, their dry masses are measured and then they were packed in plastic bags so they maintain uniform moisture content near zero. The experimental measurements will be performed on these dry samples.

\subsection{Measurement of apparent density}

From the knowledge of dimensions and masses of the four samples, the apparent density of each one can be easily determined.

The density of the granular cork is determined by the water volume variation method: a weighted quantity of granular cork is filled in a vessel containing a known water volume; the water volume increase corresponds to the volume of impregnated cork, so the density of granular cork can be deduced.

\subsection{Transient Hot Plate method for thermal effusivity and thermal capacity measurement}

The thermal effusivity is measured using the Hot Plate transient method. Contrary to the classical and symmetrical transient Hot Plate method [4] which required two similar samples; we used here the recent asymmetrical experimental device [1] (represented in Figure 2) that allows us to characterize materials by using only one sample. A plane heating element having the section $10 \times 10 \mathrm{~cm}^{2}$ is placed under the sample. A type $\mathrm{K}$ thermocouple made with two wires with a $0.005 \mathrm{~mm}$ diameter is stuck on the lower face of the heating element. This disposal is placed between two extruded polystyrene blocks with a thickness $5 \mathrm{~cm}$ set between two aluminum blocks with a thickness $4 \mathrm{~cm}$. A heat flux step is sent into the heating element and the transient temperature $T(t)$ is recorded. Since the thermocouple is in contact with polystyrene that is a deformable material, the presence of the thermocouple does not increase the thermal contact resistance between the heating element and the polystyrene. Furthermore, since polystyrene is an insulating material, this thermal resistance will be neglected. The system is modeled with the hypothesis that the heat transfer remains unidirectional (1D) at the center of the sample during the experiment.

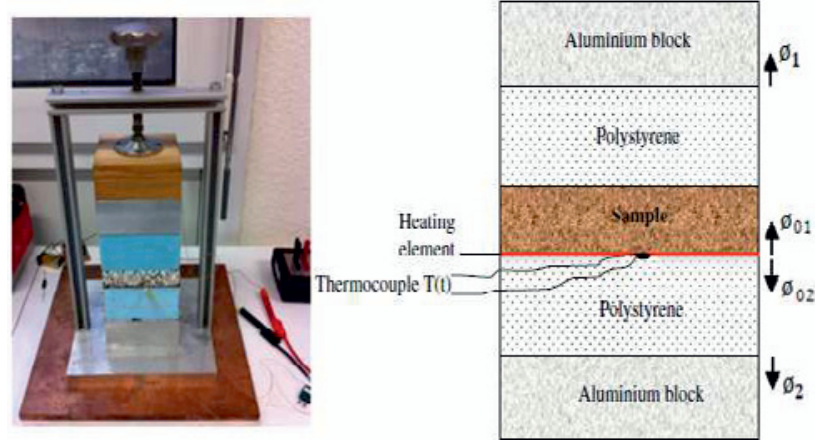

Fig. 2. View and schema of the experimental Hot Plate device.

Considering the very low value of the heat flux reaching the aluminum blocks through the polystyrene and their high thermal capacity, their temperatures are supposed equal and constant.

Within these hypotheses, one can write:

$$
\begin{aligned}
& {\left[\begin{array}{c}
\theta \\
\Phi_{01}
\end{array}\right]=\left[\begin{array}{ll}
1 & 0 \\
C_{h} & 1
\end{array}\right]\left[\begin{array}{cc}
1 & R_{C} \\
0 & 1
\end{array}\right]\left[\begin{array}{ll}
A & B \\
C & D
\end{array}\right]\left[\begin{array}{ll}
A_{i} & B_{i} \\
C_{i} & D_{i}
\end{array}\right]\left[\begin{array}{c}
0 \\
\Phi_{1}
\end{array}\right]} \\
& =\left[\begin{array}{ll}
A_{1} & B_{1} \\
C_{1} & D_{1}
\end{array}\right]\left[\begin{array}{c}
0 \\
\Phi_{1}
\end{array}\right] \\
& {\left[\begin{array}{c}
\theta \\
\Phi_{02}
\end{array}\right]=\left[\begin{array}{ll}
A_{i} & B_{i} \\
C_{i} & D_{i}
\end{array}\right]\left[\begin{array}{c}
0 \\
\Phi_{2}
\end{array}\right]}
\end{aligned}
$$

With:

$\Phi_{0}=\frac{\emptyset}{p}=\Phi_{01}+\Phi_{02}$

$\theta$ is the Laplace transform of the temperature $T(t), \Phi_{0 I}$ the Laplace transform of the heat flux density living the heating element (upstream), $\Phi_{02}$ the Laplace transform of the heat flux density living the heating element (downstream), $\Phi_{0}$ the Laplace transform of the total heat flux density produced in the heating element, $\varnothing_{0}$ the heat flux density produced in the heating element, $C_{h}$ the thermal capacity of the heating element per area unit: $C_{h}$ $=\rho_{h} c_{h} e_{h} ; R c$ the thermal contact resistance between the heating element and the sample, $\Phi_{l}$ the Laplace transform of heat flux density input on the upper aluminum block, $\Phi_{2}$ is the Laplace transform of heat flux density input on the lower aluminum block.

$$
\begin{aligned}
& A=D=\cosh \left(\frac{\rho c}{E} e \sqrt{p}\right) ; B=\frac{\sinh \left(\frac{\rho c}{E} e \sqrt{p}\right)}{E \sqrt{p}} \\
& C=E \sqrt{p} \sinh \left(\frac{\rho c}{E} e \sqrt{p}\right) \\
& A_{i}=D_{i}=\cosh \left(\sqrt{\frac{p}{a_{i}}} e_{i}\right) ; B=\frac{\sinh \left(\sqrt{\frac{p}{a_{i}}} e_{i}\right)}{\lambda_{i} \sqrt{\frac{p}{a_{i}}}} \\
& C_{i}=\lambda_{i} \sqrt{\frac{p}{a_{i}}} \sinh \left(\sqrt{\frac{p}{a_{i}}} e_{i}\right)
\end{aligned}
$$


$E$ the sample thermal effusivity, $\rho c$ the sample thermal capacity, $e$ the sample thickness, $\lambda_{i}$ the Polystyrene thermal conductivity, $a_{i}$ the Polystyrene thermal diffusivity, $e_{i}$ the Polystyrene thickness.

Combining those five equations, the system leads to:

$\theta(p)=\frac{\Phi_{0}(p)}{\frac{D_{1}}{B_{1}}+\frac{D_{i}}{B_{i}}}$

The principle of the method is to estimate the value of the parameters $E, \rho c, R c$ and $C_{h}$ that minimize the sum of the quadratic error $\Psi=\sum_{j=0}^{N}\left[T_{\text {exp }}\left(t_{j}\right)-T_{\bmod }\left(t_{j}\right)\right]^{2}$ between the experimental curve and the theoretical curve calculated with relation (8). The inverse Laplace transformation is realized by use of the De Hoog algorithm [5].

\section{Results and discussions}

\subsection{Apparent density results}

The density measurements of the four samples were made by weighing each one and knowing their dimensions. For the granular cork, it was made using the water volume variation method. The results are shown in Table 1.

Note first that the granular cork size have no effect on density of the mixture, the negligible variation observed is due to the elaboration process. The essential and practical observation is the important decay of density of white cement when it mixed with granular cork: the mixture is approximately two times lighter than white cement without cork.

Table 1. Apparent density results of the studied samples.

\begin{tabular}{|l|l|l|l|}
\hline Samples & $\begin{array}{l}\text { Masses } \\
(\mathrm{g})\end{array}$ & $\begin{array}{l}\text { Volume } \\
\left(\mathrm{m}^{3}\right)\end{array}$ & $\begin{array}{l}\text { Density } \\
\left(\mathrm{kg} \cdot \mathrm{m}^{-3}\right)\end{array}$ \\
\hline co-wc $5-6.3 \mathrm{~mm}$ & 175.3 & 0.0002 & 876.5 \\
\hline co-wc $6.3-8 \mathrm{~mm}$ & 172.3 & 0.0002 & 861.5 \\
\hline co-wc $8-12.5 \mathrm{~mm}$ & 164.3 & 0.0002 & 821.5 \\
\hline white cement & 314.2 & 0.0002 & 1571 \\
\hline
\end{tabular}

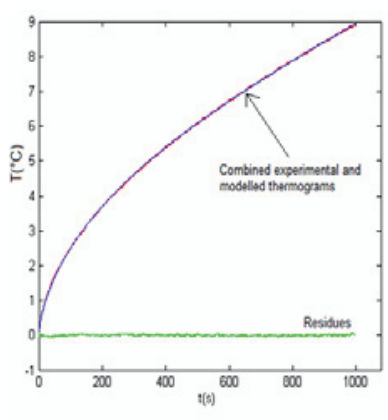

(a)

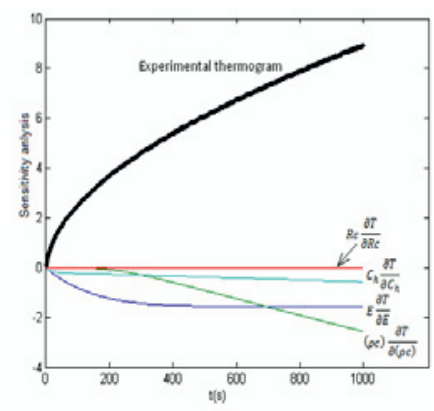

Fig. 3. (a): Experimental and modelled temperature curves (b): reduced sensitivity curves of fitting parameters

\subsection{Thermal effusivity and thermal capacity results by the asymmetrical Hot Plate method}

The asymmetrical transient Hot Plate method was applied for each sample to calculate the experimental results of characterization. For each obtained thermogram by the experiment, the Levenberg-Marquard method [6] was applied to identify the parameters $\left(E, \rho c, R c\right.$ and $\left.C_{h}\right)$ which minimize the sum of the quadratic error between the experimental curve and the theoretical curve.

Figure 3 (a) shows the curves of the two thermograms with their residues a function of time, and Figure 3 (b) shows the reduced sensitivity curves $\left(E \frac{\partial T}{\partial E} ;(\rho c) \frac{\partial T}{\partial(\rho c)} ; R c \frac{\partial T}{\partial R c} ; C_{h} \frac{\partial T}{\partial C_{h}}\right) \quad$ related to the identification parameters.

Throughout the experience, the modelled curve is with a good agreement with the experimental thermogram, this shows the validity of the hypothesis stipulating that the heat transfer remains unidirectional (1D) at the centre of the sample during the experiment.

The sensitivity curves of fitting parameters show that the thermogram, at $1000 \mathrm{~s}$, is enough sensitive to the parameters $(E)$ and $(\rho c)$; but it sensitivity to the two other parameters is very low, this implies that the thermal contact resistance and the heat capacity of heating element have no influence on the thermogram.

Table 2 shows the thermal effusivity and thermal capacity identification results. Thermal effusivity of the mixture is two times lower than that of white cement without cork, the presence of cork in the composite permits to give a less effusive material, but it decrease the thermal capacity of the mixture compared to that of white cement. Also, thermal conductivity value was deducted given that:

$E=\sqrt{\lambda \rho c}$

Thermal conductivity of the new composite is approximately three times lower than that of white cement.

Finally, note that granular cork size has no influence on thermal properties of the mixture.

Table 2. Results of identified thermal properties.

\begin{tabular}{|l|l|l|l|}
\hline Samples & $\begin{array}{l}\mathrm{E} \\
\left(\mathrm{J} \cdot \mathrm{m}^{-2} \cdot \mathrm{K}^{-1}\right. \\
\left.. \mathrm{s}^{-1 / 2}\right)\end{array}$ & $\begin{array}{l}\rho \mathrm{J} \\
\left(\mathrm{J} \cdot \mathrm{m}^{-3} \cdot \mathrm{K}^{-1}\right)\end{array}$ & $\begin{array}{l}\lambda \\
\left(\mathrm{J} \cdot \mathrm{m}^{-1} \cdot \mathrm{K}^{-1}\right)\end{array}$ \\
\hline co-wc 5-6.3 mm & 672 & 1466221 & 0.308 \\
\hline co-wc $6.3-8 \mathrm{~mm}$ & 666 & 1450340 & 0.306 \\
\hline co-wc 8-12.5 mm & 658 & 1441628 & 0.300 \\
\hline white cement & 1517 & 2655604 & 0.86 \\
\hline
\end{tabular}

\section{Conclusion}

This paper presents an experimental study characterizing the thermal properties of a new composite material based on granular cork, in order to valorize its thermal insulation, its storage capacity and its lightness compared with those of white cement without cork. The study and comparisons allow deducing that the new 
composite material is two times lighter than white cement and its insulating property is three times better than white cement without cork.

\section{References}

1. H. Bal, Y. Jannot, N. Quenette, A. Chenu, S. Gaye. Construction and Building Materials 31 (2012)

2. A. Khabbazi, N. Lahraher, O. Terahmina, Physical \& Chemical News (novembre 2003)

3. A. Khabbazi, A. Garoum, O. Terahmina, Journal Advanced of Modelling and Simulation 74 (7) (2005)

4. Y. Jannot, V. Felix, A. Degiovanni. Meas Sci Technol 21(3) 2010

5. FR. De Hoog. Soc Ind Appl Math 3(3) 1982

6. D. Marquardt. Journal on Applied Mathematics 11(2) (1963) 